\title{
RESPONSABILIDAD SOCIAL CORPORATIVA EN LAS PÉRDIDAS Y DESPERDICIOS DE ALIMENTOS EN MÉXICO
}

\author{
CORPORATE SOCIAL RESPONSIBILITY IN FOOD LOSSES AND FOOD WASTE IN \\ $M E X I C O$
}

\author{
Prof. Dr. Genaro Aguilar Gutiérrez \\ Instituto Politécnico Nacional (IPN México)
}

Resumen: El objetivo de esta investigación es desarrollar un índice de pérdidas y desperdicios de alimentos e investigar el grado de compromiso de las empresas mexicanas para disminuir los desechos de alimentos. El índice muestra que al menos 35.3\% de los alimentos se pierden o desperdician anualmente en México, un país con más de 19 millones de personas en condiciones de pobreza e inseguridad alimentaria. Se aplicó una encuesta a 3 grupos de empresarios, los resultados muestran que $23 \%$ de las grandes empresas mexicanas desarrolla cotidianamente actividades contra el desperdicio de alimentos, pero $79 \%$ de las medianas empresas y $96 \%$ de las pequeñas y micro empresas no tienen ningún plan ni realizan actividades cotidianas contra las pérdidas y desperdicios de alimentos. Proponemos la tarea de reducir desechos de alimentos como una gran área de oportunidad para la Responsabilidad Social Corporativa en México.

Palabras clave: Pérdidas y desperdicios de alimentos; Seguridad alimentaria y pobreza, Responsabilidad Social Corporativa; México.

\begin{abstract}
The objective of this research is to develop an index of food losses and food wastes and to investigate the degree of commitment of Mexican companies to reduce food waste. The index shows that at least 35.3 percent of food is lost or wasted annually in Mexico, a country with more than 19 million people living in poverty and food insecurity A survey was applied to 3 groups of entrepreneurs, the results show that $23 \%$ of large Mexican companies develop daily activities against food waste, but $79 \%$ of medium-sized enterprises and $96 \%$ of small and micro-enterprises have no plans or daily activities against food losses and food waste. We propose the task of reducing food waste as a great area of opportunity for the Corporate Social Responsibility in Mexico.
\end{abstract}

Keywords: Loss and waste of food; Food Security and Poverty, Corporate Social Responsibility; Mexico.

\footnotetext{
${ }^{1}$ Profesor titular del Instituto Politécnico Nacional (México). Tiene experiencia en Economia, con énfasis en Desarrollo Económico. Trabaja con los siguientes temas: Pobreza en México, Desarrollo económico, Federalismo fiscal, Evasión fiscal y Política social.E-mail:genaroaguilargtz@gmail.com
} 


\section{INTRODUCCIÓN}

Cada año, aproximadamente un tercio de la comida producida mundialmente para consumo humano -aproximadamente 1,300 millones de toneladas- es desechada (FAO, 2011). ${ }^{2} \quad$ Los consumidores de los países ricos desperdician casi la misma cantidad de alimentos (222 millones de toneladas) que la producción total de África Subsahariana (230 millones de toneladas).

De acuerdo con el estudio clásico de la Organización de las Naciones Unidas para la Alimentación y la Agricultura (FAO, 2012), la cantidad de comida desperdiciada anualmente en el mundo equivale a más de la mitad de la cosecha anual de cereales. La pérdida de comida también significa un gran desperdicio de otros recursos, como agua, tierra, energía, mano de obra y capital, además de la emisión de gases de efecto invernadero que contribuyen al calentamiento global y al cambio climático.

En países menos desarrollados, las pérdidas de alimentos ocurren especialmente en las etapas de producción y distribución de alimentos, normalmente por problemas logísticos, restricciones financieras, técnicas inadecuadas de administración de las cosechas y por infraestructura inadecuada para almacenamiento. En países de ingresos per cápita altos, los alimentos son desperdiciados principalmente en etapas más avanzadas de la cadena alimentaria. A diferencia de lo que ocurre en países menos desarrollados, el comportamiento de los consumidores tiene un papel enorme en los países industrializados (FAO, 2012).

Gunders (2012) calculó que, en los Estados Unidos, alrededor de 40\% de toda la comida es desperdiciada todos los años. Para un hogar promedio, esto significa un costo de

\footnotetext{
${ }^{2}$ Las definiciones de pérdidas y desperdicios de alimentos varían. La pérdida de alimentos se refiere a una disminución de la masa o la calidad nutricional de los alimentos originalmente destinados al consumo humano. Los desperdicios son los desechos de alimentos, los alimentos aptos para el consumo humano que son descartados o estropeados en el comercio minorista, el servicio de alimentos y el consumo (REICH \& FOLEY, 2014).
} 
US\$2,275 dólares anuales. Aproximadamente la mitad de agua usada en la producción de comida también es desperdiciada.

Un estudio reciente muestra que en Europa se pierden o se desperdician 720 kilocalorías per cápita al día, lo que equivale a 29\% de los alimentos disponibles en toda la Unión Europea (KUMMU et al, 2012). Ese estudio muestra, también, que para el conjunto de Norteamérica y Oceanía las pérdidas diarias por habitante son de 1,334 kilocalorías, equivalentes al 32\% de la oferta de alimentos; en tanto que en América Latina el total de pérdidas y desperdicios de alimentos es de 693 kilocalorías per cápita al día, que corresponden al 25\% de la producción de alimentos para consumo humano (KUMMU et al, 2012)

La FAO (2012, p. 1) señala que "La cuestión de las pérdidas de alimentos es muy importante en los esfuerzos para combatir el hambre, aumentar los ingresos y mejorar la seguridad alimentaria en los países más pobres del mundo". Investigaciones de todo el mundo han mostrado que la producción mundial diaria de alimentos es suficiente en cantidad para alimentar a toda la población del planeta (FAO, 2015; FAO, 2013; LIU, 2013; KUMMU et al., 2012; FAO, 2011; GOOCH et al., 2010). Sin embargo, muchos sufren hambre, evidenciando que el principal problema es la falta de acceso a los alimentos.

En el caso de México, el problema de la pobreza extrema y de su manifestación más dramática, el hambre, se genera en gran medida por la enorme desigualdad social que provoca un difícil acceso de los más pobres a los alimentos y les impide tener seguridad alimentaria (AGUILAR, 2000). Cálculos oficiales del Grupo Técnico de Pérdidas y Mermas de alimentos de la Cruzada Nacional contra el Hambre muestran que México tira a la basura el equivalente a 8,000 millones de dólares anuales; cantidad que podría servir para alimentar a cerca de 12 millones de personas o tres millones de familias, cada año. 


\section{MARCO TEÓRICO: EVOLUCIÓN DEL CONCEPTO DE RESPONSABILIDAD SOCIAL EMPRESARIAL}

Existe vasta literatura discutiendo el origen, los beneficios y las limitaciones de la Responsabilidad Social Corporativa (ZHAO, 2015; ORLITZKY, 2015; CALABRESE, MECHINI; ROSATI, 2013). Una de las conclusiones de esa discusión es que en los principios de la Sociedad Industrial y a pesar de que el concepto era bastante limitado, ya había responsabilidad social de la empresa, pero se resumía a la generación de ganancias y empleos para la sociedad. Al principio, criterios más amplios como medio ambiente y desarrollo humano no eran cuestiones relevantes; en el pensamiento gerencial de ese momento, las acciones sociales deberían ser ejercidas por el Estado, mientras que las empresas deberían buscar únicamente la maximización de las ganancias, la generación de empleos y el pago de impuestos al Gobierno. Esa visión se prolongó hasta las décadas de 70 y 80 del siglo $\mathrm{XX}$.

Una famosa cita de Milton Friedman, Premio Nobel de Economía 1976, confirma lo anterior:

Últimamente un punto de vista específico ha obtenido cada vez más aceptación: el de que los altos ejecutivos de las grandes empresas y los líderes laborales tienen una responsabilidad social más allá de los servicios que deben prestar a los intereses de sus accionistas o de sus miembros. Ese punto de vista muestra una concepción fundamentalmente equivocada del carácter y de la naturaleza de una economía libre. En tal economía solo hay una responsabilidad social del capital: usar sus recursos y dedicarse a actividades destinadas a aumentar sus ganancias hasta donde permanezca dentro de las reglas del juego, lo que significa participar en una competencia libre y abierta, sin engaños o fraude" (FRIEDMAN, 1962, p. $133)$.

Así, las empresas debían producir con eficiencia bienes y servicios y dejar la solución de las cuestiones sociales a los órganos gubernamentales competentes, ya que los gerentes de las empresas no tienen forma de determinar la urgencia de los problemas sociales ni la cantidad de recursos que debe ser empleada en la solución de tales cuestiones 
(ORLITZKY, 2015). En esa época, se pensaba que los recursos naturales serían infinitos, sin imponer restricciones a la producción y que el libre mercado sería capaz de maximizar el bienestar social. Como la teoría convencional trataba sólo de distribución eficiente de los recursos, variables como el medio ambiente no eran incorporadas a los modelos económicos (ZHAO, 2015; HULT, 2011; FERRELL et al, 2010). No obstante, la transformación del proceso productivo derivada de la revolución tecnológica y de la aplicación de la ciencia en la organización y gestión empresarial, contribuyó a la ampliación de la discusión del concepto de responsabilidad social corporativa debido a las externalidades negativas generadas por la actividad industrial (ZHAO, 2015; CARROLL; SHABANA, 2010; GARDBERG; FOMBRUN, 2006).

En una primera fase, las grandes empresas cobraron conciencia de la necesidad de tener una contribución más efectiva para la solución de los efectos secundarios indeseados de la industrialización. De esta forma, además de generar empleos, maximizar las ganancias y pagar impuestos, las empresas empezaron a cumplir con algunas exigencias legales en lo que se refiere al cuidado del medio ambiente y a las relaciones laborales. Así, el concepto de responsabilidad social corporativa incorporó algunos anhelos de la sociedad y empezó a ser comprendido en una forma más amplia (DAVIS, 1960).

En una segunda fase, las empresas buscaron integrar la función de control ambiental a sus funciones gerenciales para que todo el proceso productivo pudiera ser supervisado; así surgió la Gestión Ambiental Empresarial. De acuerdo con Barbieri (2011, p. 110), “muchos inversionistas hoy consideran las cuestiones ambientales en sus decisiones, pues saben que los pasivos ambientales están entre los principales factores que pueden corroer la rentabilidad y el patrimonio de las empresas".

Más recientemente la creciente valoración de prácticas socialmente responsables, especialmente por parte de los consumidores, provocó el tratamiento diferenciado de las inversiones sociales desde empresarios y accionistas (ZHAO, 2015). En lugar de filantropía, empezaron a practicar Responsabilidad Social Corporativa (RSC). Bajo este 
concepto, la empresa no pierde su finalidad de generar ganancias. Hoy se comprende, cada vez más, que las inversiones sociales actúan favorablemente en la medida en que la posición socialmente responsable es valorada y la socialmente irresponsable, penalizada. Las inversiones sociales atraen consumidores más atentos y más fieles, promueven trabajadores más comprometidos y vuelven la relación con el gobierno más afable. Todo esto conjuntamente se traduce en mayor productividad y mayores ganancias.

De esta forma, la práctica de la RSC se combina con políticas dirigidas a la ganancia, traduciéndose en un diferencial en términos de competitividad. Es entonces una innovación que trae posibilidades de gerenciamiento de los recursos de la empresa buscando incrementos de productividad. Si los valores dados por la cultura de la responsabilidad social pueden ser una suma de motivaciones para inversiones empresariales socialmente responsables, no podemos dejar de condicionar este comportamiento a la búsqueda de ganancias. Todo indica que el papel de la empresa no se agota, como imaginaba Friedman, con el pago de impuestos y la generación de empleos. En función de las diversas transformaciones de las prácticas sociales de las empresas, los públicos que están alrededor de ellas no lo entienden de esa forma y, en consecuencia, las empresas han debido cambiar su punto de vista.

Así, el concepto de Responsabilidad Social Corporativa (RSC) ha evolucionado. A partir de la valorización de las prácticas socialmente responsables por parte de los stakeholders (especialmente los consumidores), las prácticas de RSC hacen la diferencia en la competencia empresarial, siendo vistas como inversiones.

En el caso de América Latina, un interesante estudio muestra que la Responsabilidad Social Empresarial asume diferentes áreas de influencia y significado, a comparación de lo que pasa en Europa. Pero, en especial, la competencia y los motivadores para lograr una RSE son más fuertes y están más consolidados en Europa en comparación con América Latina. Por ejemplo, la aplicación de las leyes, la exposición a la competencia y a la globalización, los mercados financieros desarrollados, una sociedad civil más activa y 
compradores y consumidores "educados", han llevado a otro nivel la RSE en Europa y no así en América Latina (VIVES, 2014). De igual forma, Lázaro \& Gremaud (2016) argumentan que tanto las cuestiones regulatorias como el grado de avance en cuestiones sociales clave (como educación, desigualdad y pobreza) contribuyen a que tanto en Brasil como en México la Responsabilidad Social Corporativa está aún en una etapa emergente. A pesar de ello, en Brasil la RSC en relación a cuestiones de medio ambiente ha avanzado más que en México.

En general, una empresa socialmente responsable está interesada en conducir sus procesos de producción y todo lo que a ellas está directa o indirectamente relacionado de forma sustentable, minimizando agresiones al medio ambiente, favoreciendo el bienestar de la comunidad y valorizando sus recursos humanos (ZHAO, 2015). Además de la adopción de estas prácticas, una empresa socialmente responsable debe exigir el mismo compromiso de los otros individuos o instituciones con los que se relaciona, estén ligados o no a su actividad económica principal. En la práctica, en la medida en que este comportamiento es implementado, se traduce en mayores mercados, consumo, ganancias e inversiones.

Sin embargo, no podemos decir que el desarrollo de las acciones socialmente responsables deriva exclusivamente de la reestructuración de las estrategias de competencia. La incorporación de la cultura de responsabilidad social en las empresas tiene importancia como componente ideológico de las inversiones privadas. Las firmas, como estructuras celulares del proceso productivo, al incorporar la cultura de la responsabilidad social entienden que su papel social debe exceder sus actividades productivas y el pago de impuestos, más aún en la medida en que se vuelven extremadamente poderosas. De esta forma, las grandes empresas, especialmente en los países desarrollados, han venido buscando una responsabilidad acerca de los stakeholders mayor que aquella que históricamente les pertenece.

En el campo de la protección al medio ambiente, el surgimiento de diversas iniciativas ayudó a consolidar la visión de la responsabilidad social empresarial a partir de 
la década de 1990. Entre ellas podemos mencionar la realización de la Conferencia de las Naciones Unidas sobre Medio Ambiente, Río 1992, el desarrollo del GRI (Global Reporting Initiative) y las Metas de Desarrollo del Milenio. Todas estas iniciativas reflejaron un cambio cultural en el comportamiento de la sociedad y de las empresas y contribuyeron a la diseminación de la práctica de la Responsabilidad Ambiental y Social Corporativa. Sin embargo, la agenda está inconclusa.

\subsection{PÉRDIDAS Y DESPERDICIOS DE ALIMENTOS}

FAO (2012) define pérdidas pos-cosecha como cualquier reducción en la disponibilidad de alimentos para el consumo, mismas que se presentan durante la manipulación, transporte, almacenamiento, industrialización o comercialización de los productos agropecuarios. Esas pérdidas son un problema antiguo para el hombre, que necesitó desarrollar tecnologías para la preservación de alimentos, principalmente en las regiones del mundo condiciones climatológicas extremosas.

La minimización de pérdidas o desperdicios de alimentos tiene varias ventajas, tales como un aumento significativo de la oferta de alimentos sin aumentar las áreas de cultivo, ahorros de energía invertida para producir y comercializar los alimentos perdidos, reducción de la contaminación debido a la disminución de la materia orgánica en descomposición y una mejor satisfacción de las necesidades de los consumidores así como una mejor nutrición con la misma cantidad de energía, tierra, agua y trabajo.

Un exhaustivo estudio desarrollado en el año 2011 y publicado en 2012 por la Organización de las Naciones Unidas para la Alimentación y la Agricultura (FAO) indica que un tercio de los alimentos producidos para el consumo humano se pierde o se desperdicia

a nivel mundial, y equivale aproximadamente a 1,300 millones de toneladas por año. 
El estudio de la FAO (2012) también muestra que en términos per cápita, se desperdicia mucho más comida en el mundo industrializado que en los países de menor desarrollo. Las estimaciones de dicho estudio sugieren que los desperdicios per cápita de alimentos de los consumidores en Europa y América del Norte es de 95 a $115 \mathrm{~kg}$ por año, mientras que esta cifra en el África subsahariana y el sur/sudeste asiático sólo es de $6.11 \mathrm{~kg}$ por año. Sin embargo, los desperdicios son muy grandes también en los países de América Latina y el Caribe y llegan a $65 \mathrm{~kg}$ per cápita.

Las causas de las pérdidas y desperdicios de alimentos en los países de bajos ingresos están conectadas principalmente a problemas financieros, de cultura empresarial y a las limitaciones técnicas en las fases de cosecha y almacenamiento (especialmente por falta de inversión en instalaciones y transporte con refrigeración en zonas con climas tropicales o semi-tropicales), a la falta de infraestructura de envasado y de sistemas modernos de comercialización.

Así, el estudio de la FAO arguye que, teniendo en cuenta que muchos de los pequeños agricultores en los países de menor desarrollo viven en los márgenes de la inseguridad alimentaria, la reducción de las pérdidas de alimentos podría tener un impacto inmediato y significativo sobre sus niveles de vida.

Las pérdidas pos-cosecha de alimentos ocurren en función de la depreciación de la calidad de los productos, debido al deterioro causado principalmente por manipulación inadecuada de los alimentos y por la acción de agentes externos como plagas y enfermedades. Los principales factores que determinan esas depreciaciones son incorrecta manipulación de los alimentos y malas prácticas de transporte y almacenamiento (FAO, 2012).

Las causas primarias de pérdidas de alimentos, aquellas que afectan directamente el alimento, son clasificadas en los siguientes grupos: a) biológicas, b) microbiológicas, c) químicas, d) reacciones bioquímicas, e) mecánicas, f) físicas, g) físiológicas y h) 
psicológicas. Los daños mecánicos, determinados por diferentes tipos de lesiones (cortes, rupturas, caídas y deformaciones) son el principal motivo de pérdidas pos-cosecha para muchos frutos y hortalizas y se presentan, principalmente, por manipulación inadecuada del producto, en cualquier etapa de su período de vida, desde la cosecha hasta su comercialización. Por tanto, prácticas empresariales dirigidas a la preparación de mano de obra especializada tendrían efectos remediales en el problema.

Un gran número de productos hortícolas presenta problemas de daños mecánicos. Algunas frutas y hortalizas, por tener un elevado contenido de agua y estructura muy delicada, como la pera y la papaya, por ejemplo, acaban siendo altamente susceptibles a todo tipo de daños. Otros productos son más resistentes, pero por el hecho de ser sometidos al transporte y al almacenamiento por largos períodos de tiempo, afecta la preservación de su integridad física (SOUZA; HENZ; PEIXOTO, 2003). Los daños causados en los tejidos modifican la actividad fisiológica de los alimentos. Dichos daños provocan, frecuentemente, el rompimiento de las células de la epidermis, causando el desencadenamiento de reacciones enzimáticas que promueven el surgimiento de compuestos de coloración marrón, responsables de la depreciación de los alimentos (DURIGAN, 2001; RADI et al., 1997; SAMIM; BANKS, 1993). Tales daños provocan también el aumento de enfermedades pos-cosecha, el aumento en la tasa de respiración y en la síntesis de etileno, además de alteraciones en el color, aroma, sabor y textura; todo lo cual vuelve a los productos más perecederos. 


\subsection{RESPONSABILIDAD SOCIAL CORPORATIVA Y DESPERDICIOS DE ALIMENTOS}

Algunos autores afirman que las pérdidas y desperdicios de alimentos pueden ser reducidas a un nivel aceptable si todo el sistema de manipulación es modificado desde la cosecha hasta el consumidor (HERTOG, 2007). Sin embargo, lo que se observa es que desde el punto de vista empresarial esas pérdidas son frecuentemente descuidadas (HARTMAN, 2011). Además de ello, otros factores técnicos que deben ser resueltos tecnológicamente por las empresas; deben ser considerados. Las hortalizas, por ejemplo, presentan una elevada tasa de transpiración con un consecuente y rápido deterioro fisiológico, principalmente en ambientes con temperatura elevada (HERTOG, 2007). Esa característica normalmente es ignorada por los comercializadores de alimentos frescos, dado que tales productos son vendidos directamente del productor al minorista sin ningún proceso de congelación u otro tratamiento que tenga como objetivo aumentar el tiempo de vida de los alimentos. Así, por la deficiencia en la manipulación y la ausencia de condiciones adecuadas de almacenamiento, las pérdidas de alimentos son muy grandes (DURIGAN, 2001).

En los países con menos desarrollo, la elaboración de planes para la reducción de pérdidas y desperdicio de productos alimenticios no siempre es apropiada y su aplicación raramente es exitosa, ya que el producto fresco o perecedero es frecuentemente manipulado de la misma forma que el duradero. Eso ocurre debido a que las soluciones para muchos problemas de manipulación, embalaje y almacenamiento están relacionadas a factores educativos y sociológicos. Así, cualquier plan para reducción de pérdidas de alimentos debe ser fundamentado en cambios culturales y estructurales a lo largo de la cadena de comercialización de los alimentos. En este proceso donde debe jugar un papel fundamental la responsabilidad social corporativa. 
Las pérdidas y desperdicios de alimentos generan graves consecuencias económicas y sociales, provocan una gran variación en el comportamiento del mercado, induciendo cambios en importantes parámetros económicos. La existencia de pérdidas de alimentos hace que la cantidad de equilibrio del mercado disminuya y el precio aumente. Cuando el precio de equilibrio de mercado crece, se reduce el excedente del consumidor. En este caso, el consumidor paga el costo de las pérdidas y de los desperdicios que es incluido en el precio final del producto. De forma general, cualquier nivel de pérdidas perjudica a los consumidores (CAIXETA, 2009).

En términos económicos, la reducción de las pérdidas de alimentos causa un aumento de los ingresos totales del distribuidor minorista (retail seller), quien de esa forma puede ofrecer un mayor volumen de alimentos. Ese incremento provoca, a su vez, un aumento en el consumo proporcionalmente mayor que la reducción en la cantidad ofertada debido a las pérdidas. En ese sentido, la diferencia entre las elasticidades-precio de la oferta y de la demanda mide los beneficios de la reducción de las pérdidas para la sociedad (COSTA; CAIXETA, 2013).

Las pérdidas de alimentos tan indeseadas socialmente pueden ser reducidas simplemente mediante el combate a los factores que las provocan. De esta forma, entre los factores responsables de las pérdidas de alimentos están, por ejemplo, embalajes inadecuados. Pero las pérdidas causadas a la sociedad por las pérdidas y desperdicios de alimentos pueden ser, en su mayor parte, debidos a las formas de gestión y manipulación de los productos en la red minorista (COSTA; CAIXETA, 2013). Para que las pérdidas y desperdicios se reduzcan es necesario entonces una amplia gama de tareas, tanto desde las empresas como desde los consumidores.

Así, la responsabilidad social de los empresarios del sector de alimentos debe ser comprendida como una forma más amplia de accountability hacia la sociedad. La responsabilidad de las empresas está relacionada con un conjunto de factores: a) consumo, desde la empresa, de los recursos naturales propiedad de la humanidad; b) consumo, desde 
la empresa, de los capitales financieros y tecnológicos debido al uso de la capacidad de trabajo y producción que pertenece a personas físicas, integrantes de la sociedad; y c) apoyo que reciben las empresas por los Estados nacionales, en sus actividades cotidianas (COSTA; PEIXOTO, 2013).

En la medida en que las empresas del sector de alimentos (producción y distribución) están empleando cuantiosos recursos naturales y humanos, y parte importante de dichos recursos está siendo desperdiciada por medio del desecho cotidiano de alimentos, esta situación debería llamar la atención de las empresas y transformarse en un eje de la Responsabilidad Social Corporativa.

\section{MÉTODO}

Fueron usados dos instrumentos. Por un lado se construyó un índice de desperdicio de alimentos y también se aplicó una encuesta para medir responsabilidad social en materia de pérdidas de alimentos, en una muestra de 278 empresas que sí respondieron al cuestionario. La encuesta fue aplicada a partir de un muestreo estratificado aleatorio, se aplicó un cuestionario y la tasa de respuesta fue de 92\% (302 empresas era el universo inicial). Los cuestionarios fueron aplicados durante los meses de agosto y noviembre del año 2015 y el cuestionario se aplicaba siempre que se tuviera como entrevistado al tomador de decisiones en responsabilidad social de la empresa.

\subsection{EL ÍNDICE DE DESPERDICIO DE ALIMENTOS}

Definimos el Consumo Nacional Aparente de cualquier alimento de la siguiente manera:

$$
A D C_{i, t}=Y_{i, t}-X_{i, t}+M_{i, t}
$$

Donde:

$\mathrm{ADC}_{\mathrm{i}, \mathrm{t}}=$ es el consumo nacional aparente del producto $\mathrm{i}$, en el período t;

$\mathrm{Y}_{\mathrm{i}, \mathrm{t}}=$ es la producción nacional del producto $\mathrm{i}$ en el período $\mathrm{t}$;

$\mathrm{X}_{\mathrm{i}, \mathrm{t}}=$ Es la exportación nacional del producto i en el período $\mathrm{t}$;

$\mathrm{M}_{\mathrm{i}, \mathrm{t}}=$ son las importaciones nacionales del producto $\mathrm{i}$ en el período $\mathrm{t}$ 
Para cualquier producto i (tomate, huevo, carne, frijol, arroz, etc) se obtendrá, mediante esta fórmula, el Consumo Nacional Aparente en el año t.

Sin embargo, a esa cantidad debemos restar el producto desperdiciado en la cadena productor-consumidor, para obtener el consumo real y el desperdicio:

$$
W_{i, t}=A D C_{i, t}-R C_{i, t}
$$

Donde:

$\mathrm{W}_{\mathrm{i}, \mathrm{t}}=$ es el desperdicio nacional del producto $\mathrm{i}$ en el período $\mathrm{t}$;

$\mathrm{RC}_{\mathrm{i}, \mathrm{t}}=$ es el consumo real del producto $\mathrm{i}$, en el período $\mathrm{t}$

Podemos obtener una aproximación al Consumo real de cada producto:

$$
R C_{i, t}=c_{i, t}^{1}+c_{i, t}^{2}+c_{i, t}^{3}+\ldots+c_{i, t}^{4}
$$

Es decir la cantidad consumida de un producto es la suma de las cantidades consumidas dentro de los hogares, restaurantes, hoteles, escuelas, hospitales, empresas, etc.

$$
R C_{i, t}=\sum_{i=1}^{n} c_{i, t}
$$

Sustituyendo (4) en (2) tenemos:

$$
W_{i, t}=A D C_{i, t}-\sum_{i=1}^{n} c_{i, t}
$$

Esta fórmula es el índice de desperdicio del alimento i en el período $t$. Para derivar la fórmula del cálculo del desperdicio de alimentos en un país, se debe seleccionar un grupo de productos representativos de la Canasta Básica de Alimentos de ese país. 
Adicionalmente, se debe calcular el peso que cada uno de esos alimentos tiene en la dieta de las personas. La fórmula del Índice General de Desperdicio de Alimentos es:

$$
W I=\frac{W_{1, t}}{T C} \frac{\pi_{1, t}}{1}+\frac{W_{2, t}}{T C} \frac{\pi_{2, t}}{1}+\frac{W_{3, t}}{T C} \frac{\pi_{3, t}}{1}+\frac{W_{4, t}}{T C} \frac{\pi_{4, t}}{1}+\ldots+\frac{W_{k, t}}{T C} \frac{\pi_{k, t}}{1}
$$

Donde $T C$ es el consumo total de alimentos de la población

Es decir, el Desperdicio de alimentos (WI) en un país es una suma ponderada de los desperdicios de cada alimento, el factor de ponderación es la participación $(\pi)$ de cada alimento en la dieta de la población de ese país.

\subsection{ENCUESTA A EMPRESAS}

Con la finalidad de observar el grado de compromiso que las empresas tienen para contribuir a la disminución de las pérdidas y desperdicios de alimentos en México, se aplicó un survey, entre los meses de agosto y noviembre del año 2015. Esta encuesta fue aplicada a 278 empresarios o ejecutivos mexicanos, agrupados en tres grandes niveles: a) grandes empresas (35 empresas); b) medianas empresas (65 empresas); c) micro y pequeñas empresas (178 empresas).

Los cuestionarios fueron aplicados a ejecutivos en la muestra de las 278 empresas, buscando mantener el análisis solo con responsables de decidir sobre las acciones de responsabilidad social. La investigación utilizó cuestionarios cerrados y analizó datos sin la interferencia del entrevistador, tratando de identificar la visión de los administradores de esas empresas que forman parte del sector agroalimentario mexicano sobre las prácticas de responsabilidad social empresarial. A través del análisis cuantitativo se obtuvieron las conclusiones. 
El cuestionario se basó en el modelo propuesto por Carrol (2003) y contenía las siguientes partes: I) caracterización de la empresa y perfil de los trabajadores; II) aspectos relevantes de la empresa en relación a su actitud frente a la responsabilidad social empresarial; III) caracterización de las prácticas de responsabilidad social empresarial que la empresa ha implementado; IV) opinión sobre el desempeño de la empresa en función de los competidores y; V) opinión sobre el desempeño, relaciones y estrategias de la empresa con los diferentes stakeholders.

El cuestionario tenía 12 preguntas realizadas cara a cara. El objetivo fue evaluar la inversión social privada en México y trató de cumplir con lo siguiente:

1. Identificar cuáles empresas de México desarrollaron actividades de responsabilidad social y cómo tales empresas definen responsabilidad social;

2. Identificar si las empresas vislumbran el crecimiento o fortalecimiento de los negocios a través de actividades de responsabilidad social;

3. Identificar cuáles son el tipo de empresas que más invierten en la responsabilidad social contra las pérdidas y desperdicios de alimentos en México;

4. Mapear el tipo de actividad de responsabilidad social que cada empresa desarrolla y cuál es la segmentación más utilizada (salud, educación, inclusión social, etc.).

5. Identificar y evaluar como tales actividades influyen en la imagen institucional y en la percepción de sus stakeholders.

Para evaluar la fortaleza del instrumento de captación de información se aplicó el coeficiente alfa de Cronbach. De acuerdo con Cortina (1993), el coeficiente alfa es una de las herramientas estadísticas más importantes en investigaciones que prueban la confiabilidad de cuestionarios aplicados. Una revisión del Social Sciences Citations Index para la literatura publicada entre 1960 y 2015 muestra que el artículo de Cronbach (1951) fue citado aproximadamente 60 ocasiones por año en un total de 398 revistas científicas diferentes.

El coeficiente alfa fue descrito en 1951 por Lee J. Cronbach (CRONBACH, 1951). Es un índice utilizado para medir la confiabilidad del tipo consistencia interna de una escala; es decir, para evaluar la magnitud en que los ítems de un cuestionario están correlacionados (CORTINA,1993). En otras palabras, el alfa de Cronbach es el promedio 
de las correlaciones entre los ítems que forman parte de un instrumento (STREINER, 2003). También se puede conceptualizar este coeficiente como la medida por medio de la cual algún elemento medido está presente en cada ítem. Generalmente, un grupo de ítems que explora un factor común muestra un elevado valor alfa de Cronbach (ROGERS, SHMITI; MULLINS, 2002).

Para estimar el alfa, se considera $\mathbf{X}$ como una matriz $n \times k$ que corresponde a las respuestas cuantificadas de un cuestionario. Cada línea de $\mathbf{X}$ representa un sujeto y cada columna representa una pregunta. Las respuestas cuantificadas pueden estar en cualquier escala (LEONTITSIS; PAGGE, 2007).

Así, de acuerdo con Leontitsis y Pagge (2007), el coeficiente alfa de Cronbach es medido de acuerdo con la ecuación 7:

$$
\alpha=\frac{k}{k-1}\left[\frac{\sigma_{\tau}^{2}-\sum_{i=1}^{k} \sigma_{i}^{2}}{\sigma_{\tau}^{2}}\right]
$$

Donde $\sigma_{i}^{2}$ es la varianza de cada columna de $\mathbf{X}$, es decir, la varianza relacionada a cada pregunta de $\mathbf{X}, \mathbf{y} \sigma_{\tau}^{2}$ es la varianza de la suma de cada línea de $\mathbf{X}$, es decir, es la varianza de la suma de las respuestas de cada sujeto. También se debe observar que $k$ debe ser mayor que 1 (para evitar cero en el denominador) y $n$ debe ser mayor que 1 para que no haya cero en el denominador en el cálculo de $\sigma_{i}^{2}$ y de $\sigma_{\tau}^{2}$.

En la ecuación anterior, $k$ es un factor de corrección. Si hay consistencia en las respuestas captadas por un cuestionario, entonces $\sigma_{\tau}^{2}$ será relativamente grande; haciendo que el alfa $\alpha$ tienda a 1 .

De acuerdo con Christmann y Van Aelst (2006), el alfa de Cronbach también puede ser calculado tomando en cuenta la covarianza entre los ítems del cuestionario, con la siguiente ecuación: 


$$
\alpha=\frac{p}{p-1} \frac{\sum \sum_{j=k} \sigma_{j k}}{\sum \sum_{j, k} \sigma_{j k}}
$$

Donde $\sigma_{j k}$ es la covarianza del par $\left(\mathrm{Y}_{\mathrm{j}}, \mathrm{Y}_{\mathrm{k}}\right)$.

Interpretación de resultados. El coeficiente alfa de Cronbach es una propiedad inherente del patrón de respuesta de la población estudiada, no una característica de la escala por sí sola; es decir, el valor alfa sufre cambios de acuerdo con la población en la cual se aplica una escala (STREINER, 2003).

El valor mínimo aceptable para alfa es de 0.70; debajo de ese valor la consistencia interna de la escala utilizada es considerada bajo. En contrapartida, el valor máximo esperado es de 0.90; arriba de este valor, se puede considerar que hay redundancia o duplicación; es decir, varios ítems están midiendo exactamente el mismo elemento y por lo tanto los ítems redundantes deben ser eliminados. Usualmente, son preferidos valores alfa entre 0.80 y 0.90 (STREINER, 2003).

\section{RESULTADOS: DESPERDICIO DE ALIMENTOS}

Para el cálculo del índice de desperdicio de alimentos de México se utilizaron un conjunto de informaciones relevantes, todas del año 2014: a) producción nacional de alimentos de la Secretaría de Agricultura de México (240 conceptos genéricos); b) exportaciones e importaciones de esos alimentos; c) micro datos del consumo de alimentos en los hogares de México, contenidos en la Encuesta Nacional de Ingresos y Gastos de los Hogares ${ }^{3}$; y c) datos nacionales de consumo de alimentos fuera del hogar (realizados en

\footnotetext{
${ }^{3}$ Nota metodológica: La “Encuesta Nacional de Ingresos y Gastos de los Hogares” (ENIGH) de México es realizada cada dos años (desde 1990) por el Instituto Nacional de Estadística y Geografía (INEGI) de México. Las bases de micro datos originales de las Encuestas Nacionales de Ingresos y Gastos de los Hogares de México contienen información de gastos y características de los hogares en archivos separados. En los archivos de gasto de las personas los datos estaban presentados de tal forma que cada tipo de gasto ocupaba un registro; de esa manera, para cada individuo había tantos registros como tipos de gastos tuviera: una persona podría tener 3 ó 4 registros en caso de que tuviera, respectivamente, 3 ó 4 gastos. Los archivos de características de las viviendas presentaban información relevante de las familias, como el número de
} 
empresas, restaurantes, hoteles y escuelas), contenidos en la Encuesta Nacional de Ingresos y Gastos de los Hogares de México.

El cuadro 1 muestra que en México se desperdiciaron, en el año 2014, alrededor de 11.1 millones de toneladas de alimentos, en un grupo de 34 productos de la canasta básica de alimentos. El índice (ponderado) de desperdicio es de $35.32 \%$.

Si estos alimentos se distribuyeran entre la población que vive con carencia de alimentos en México, podrían ser entregados, cada semana, a cada familia pobre de México: 14.6 libras de tortilla; 5.5 libras de arroz; 8.9 libras de carne de res; 7.8 libras de carne de puerco; 16.06 libras de carne de pollo; 0.4 libras de atun; 2.8 libras de pescado; 0.5 libras de camarón; 17 litros de leche y 11.9 libras de huevo; 7.6 libras de papa; 4.6 libras de aguacate; 10.5 libras de jitomate; 5 libras de frijol; 6.6 libras de mango; 7.7 libras de plátano; 4.4 libras de manzana; 1.4 libras de guayaba; etc. Eso significa que la carencia de alimentos entre la población más pobre de México podría acabar.

integrantes, el factor de expansión y características socio-económicas del hogar. Todos los archivos distinguían a las personas y familias por folios o códigos clave. La forma de proceder para preparar los archivos necesarios para analizar los gastos de los hogares fue, grosso modo, la siguiente. A) A partir de los archivos de gastos se calculó el gasto de todos los individuos de cada familia, generando un nuevo archivo que contenía la información de gasto total de cada familia; B) se empató (match) este archivo (a través del folio) con el de las características de las hogares para obtener, asociado a cada registro de gasto familiar, el número de integrantes de la familia; C) en seguida, se calculó el gasto total familiar; D) A partir de este último tipo de archivos (para cada año) se calcularon los gastos, por alimento, a que nos referimos en esta investigación. 
Cuadro 1. Desperdicio de alimentos en México, por producto; año 2014.

\begin{tabular}{|c|c|c|}
\hline Alimento & Desperdicio (\%) & Toneladas \\
\hline Tortilla & 10.52 & $699,748.30$ \\
\hline Pan (productos de trigo) & 50.75 & $1,728,533.65$ \\
\hline Arroz & 52.49 & $264,670.86$ \\
\hline Carne de Res & 39.05 & $427,451.97$ \\
\hline Carne de Puerco & 45.82 & $374,684.05$ \\
\hline Carne de Pollo & 44.08 & $766,405.73$ \\
\hline Atún & 26.24 & $19,863.71$ \\
\hline Pescados y sardinas & 60.56 & $134,728.08$ \\
\hline Camarón & 42.30 & $26,024.38$ \\
\hline Leche de vaca & 41.60 & $1,700,766.37$ \\
\hline Huevo blanco y rojo & 42.18 & $571,830.37$ \\
\hline Papa & 41.56 & $361,780.48$ \\
\hline Aguacate & 60.45 & $220,053.58$ \\
\hline Ajo & 23.91 & $11,080.19$ \\
\hline Calabacita y calabaza & 16.41 & $41,532.40$ \\
\hline Cebolla & 35.93 & $265,373.77$ \\
\hline Chile & 49.44 & $436,781.05$ \\
\hline Jitomate & 32.32 & $502,428.79$ \\
\hline Nopal & 70.85 & $88,811.63$ \\
\hline Pepino & 50.92 & $54,900.91$ \\
\hline Tomate verde & 19.91 & $80,925.91$ \\
\hline Zanahoria & 21.29 & $51,137.48$ \\
\hline Frijol & 27.96 & $238,854.24$ \\
\hline Guayaba & 56.58 & $67,542.53$ \\
\hline Limón & 32.71 & $84,741.23$ \\
\hline Mango & 53.45 & $315,724.25$ \\
\hline Manzana y perón & 48.09 & $207,537.93$ \\
\hline Melón & 40.00 & $52,373.79$ \\
\hline Naranja & 22.52 & $668,028.17$ \\
\hline Papaya & 22.12 & $75,736.29$ \\
\hline Piña & 31.80 & $51,738.14$ \\
\hline Plátano verde y tabasco & 52.15 & $368,943.36$ \\
\hline Sandía & 18.86 & $42,051.37$ \\
\hline Uva & 44.16 & $54,915.50$ \\
\hline Desperdicio Nacional & 35.32 & $11,057,700.47$ \\
\hline
\end{tabular}

FUENTE: CÁLCULOS REALIZADOS CON EL ÍNDICE DE DESPERDICIOS DE ALIMENTOS. 


\subsection{RESULTADOS: VISIÓN EMPRESARIAL}

De acuerdo con los resultados globales de la encuesta aplicada a empresarios del sector en México, 23\% de las grandes empresas mexicanas desarrolla cotidianamente actividades contra el desperdicio de alimentos, pero $79 \%$ de las medianas empresas y $96 \%$ de las pequeñas y micro empresas no tienen ningún plan ni realizan actividades cotidianas contra el desperdicio de alimentos. Al evaluar los resultados obtenidos en la investigación, se observa que la mayoría de los gerentes y directores de las empresas son del sexo masculino (88\%). En cuanto a la edad, la mayor frecuencia es arriba de los 30 años (96\%). El grado de escolaridad muestra que el $53 \%$ posee estudios de licenciatura y $24 \%$ tiene estudios de posgrado.

En cuanto a la política de la empresa frente a la responsabilidad social empresarial, en la segunda parte fueron evaluadas las preferencias de las empresas estudiadas de acuerdo con su grado de importancia. La suma de sus preferencias era de 10 puntos, por lo que cada una de las prácticas empresariales fue mutuamente excluyente.

Los resultados muestran que, entre las diversas opciones, la práctica de “cumplimiento de las normas de la sociedad y de las normas éticas" obtuvo la mayor frecuencia, con un valor promedio de 3.0. La segunda práctica más importante de las empresas, con un promedio de 2.8, fue la de "cumplir con la legislación vigente". Este ítem obtuvo un alfa de Cronbach de 0.88 , lo que sugiere una muy buena formulación de las preguntas.

Al cuestionar sobre la finalidad de buscar nuevas oportunidades de negocio, "mejorar resultados financieros" (con valor de 4.1) fue el objetivo más recurrente, seguido de "mejorar la imagen de la empresa desde el punto de vista ético y moral". El alfa de Cronbach en este caso fue de 0.83 . 
Cuadro 2. La fortaleza en la administración de una empresa debe ser evaluada en función de....

\begin{tabular}{lrrrr}
\hline Alternativas & Grandes & Medianas & $\begin{array}{c}\text { Micro y } \\
\text { Pequeñas }\end{array}$ & Promedio \\
\hline La maximización de las ganancias de la & & & & \\
empresa & 2.7 & 1.8 & 3.1 & 2.5 \\
El cumplimiento de las leyes & 2.3 & 3.2 & 2.9 & 2.8 \\
La realización de acciones filantrópicas & 1.1 & 2.1 & 1.9 & 1.7 \\
El cumplimiento de las normas de la & & & & \\
sociedad y de las normas éticas & 3.9 & 2.9 & 2.1 & 3.0 \\
\hline
\end{tabular}

FUENTE: ENCUESTA A EMPRESARIOS DEL SECTOR AGROALIMENTARIO DE MÉXICO.

Cuadro 3. Es importante para la empresa buscar nuevas oportunidades de negocios para:

\begin{tabular}{|c|c|c|c|c|}
\hline & & Empresas & & \\
\hline Alternativas & Grandes & Medianas & $\begin{array}{l}\text { Micro y } \\
\text { Pequeñas }\end{array}$ & Promedio \\
\hline $\begin{array}{l}\text { Mejorar la imagen de la empresa } \\
\text { desde el punto de vista moral y ético } \\
\text { Garantizar el cumplimiento de las }\end{array}$ & 3.8 & 2.9 & 1.9 & 2.9 \\
\hline leyes & 1.2 & 0.8 & 1.8 & 1.3 \\
\hline Mejorar los resultados financieros & 3.8 & 4.4 & 4.2 & 4.1 \\
\hline $\begin{array}{l}\text { Demostrar capacidad para resolver } \\
\text { problemas sociales }\end{array}$ & 1.2 & 1.9 & 2.1 & 1.7 \\
\hline
\end{tabular}

FUENTE: ENCUESTA A EMPRESARIOS DEL SECTOR AGROALIMENTARIO DE MÉXICO.

Como se puede observar en la tabla 6, en la percepción de los entrevistados, la mejor forma de definir la responsabilidad social corporativa es "actuar en función de reglas éticas y morales" (3.5 en promedio) y "realizar acciones de voluntariado en la comunidad" (2.9). Este grupo de opciones tuvo un alfa de Cronbach de 0.82 . 
Cuadro 4. La responsabilidad social empresarial debe ser definida como:

\begin{tabular}{|c|c|c|c|c|}
\hline & & Empresas & & \\
\hline Alternativas & Grandes & Medianas & $\begin{array}{l}\text { Micro y } \\
\text { Pequeñas }\end{array}$ & Promedio \\
\hline $\begin{array}{l}\text { Hacer aquello que las leyes y la } \\
\text { comunitaria exige }\end{array}$ & 0.8 & 2.1 & 1.9 & 1.6 \\
\hline $\begin{array}{l}\text { Realizar acciones de voluntariado en } \\
\text { la comunidad }\end{array}$ & 2.1 & 3.2 & 3.3 & 2.9 \\
\hline $\begin{array}{l}\text { Actuar en función de las reglas éticas y } \\
\text { morales }\end{array}$ & 4.4 & 2.9 & 3.1 & 3.5 \\
\hline $\begin{array}{l}\text { Garantizar la mayor rentabilidad de la } \\
\text { empresa }\end{array}$ & 2.7 & 1.8 & 1.7 & 2.1 \\
\hline
\end{tabular}

FUENTE: ENCUESTA A EMPRESARIOS DEL SECTOR AGROALIMENTARIO DE MÉXICO.

El cuadro 5 muestra los resultados del origen de la responsabilidad social empresarial, de acuerdo con los entrevistados. Este ítem tuvo un alfa de Cronbach de 0.88.

Así, al cuestionar sobre la razón de la responsabilidad social empresarial, los entrevistados indicaron que era importante ya que "garantiza que la empresa cumple desde el punto de vista social y empresarial" (3.6 en promedio).

El cuadro 6 muestra los resultados al analizar a detalle lo que en la práctica significa la RSC, los ejecutivos respondieron que la misma significa "promover comportamientos morales y éticos" (3.3). La prueba de confiabilidad de las respuestas muestra un alfa de Cronbach de 0.89 para este ítem. 
Cuadro 5. La responsabilidad social empresarial es importante ya que permite:

\begin{tabular}{lcccc}
\hline & \multicolumn{2}{c}{ Empresas } & & \\
\hline Alternativas & Grandes & Medianas & $\begin{array}{c}\text { Micro y } \\
\text { Pequeñas }\end{array}$ & Promedio \\
\hline $\begin{array}{l}\text { Dar un apoyo a instituciones } \\
\text { educativas públicas o privadas }\end{array}$ & 1.4 & 1.9 & 1.6 & 1.6 \\
$\begin{array}{l}\text { Lograr un buen nivel de eficiencia } \\
\text { operativa }\end{array}$ & 0.0 & 2.6 & 1.8 & 1.5 \\
$\begin{array}{l}\text { Garantizar que la empresa cumple } \\
\text { desde el punto de vista social y } \\
\text { empresarial }\end{array}$ & & & & \\
$\begin{array}{l}\text { Reconocer y respetar las normas } \\
\text { éticas y morales adoptadas por la } \\
\text { sociedad }\end{array}$ & 4.4 & 2.7 & 3.7 & 3.6 \\
\hline
\end{tabular}

FUENTE: ENCUESTA A EMPRESARIOS DEL SECTOR AGROALIMENTARIO DE MÉXICO.

Cuadro 6. Una actitud comprometida de responsabilidad social empresarial:

\begin{tabular}{|c|c|c|c|c|}
\hline & & Empresas & & \\
\hline Alternativas & Grandes & Medianas & $\begin{array}{l}\text { Micro y } \\
\text { Pequeñas }\end{array}$ & Promedio \\
\hline Trae beneficios financieros... & 0.2 & 1.7 & 2.3 & 1.4 \\
\hline Promueve $\quad$ actividades & & & & \\
\hline $\begin{array}{l}\text { voluntariado en la comunidad } \\
\text { Garantiza el cumplimiento de las }\end{array}$ & 2.9 & 3.1 & 2.9 & 3.0 \\
\hline leyes & 2.6 & 2.1 & 2.2 & 2.3 \\
\hline $\begin{array}{l}\text { Promueve comportamientos morales } \\
\text { y éticos }\end{array}$ & 4.3 & 3.1 & 2.6 & 3.3 \\
\hline
\end{tabular}

FUENTE: ENCUESTA A EMPRESARIOS DEL SECTOR AGROALIMENTARIO DE MÉXICO.

Y para estos gestores de empresas la RSC es importante ya que "permite reconocer que no todos los medios justifican los fines". Al aplicar la prueba de consistencia del coeficiente alfa de Cronbach, se obtuvieron resultados consistentes con la hipótesis de consistencia entre las primeras 5 respuestas, en los tres grupos empresariales.

Cuadro 7. La responsabilidad social empresarial es importante ya que: 


\begin{tabular}{|c|c|c|c|c|}
\hline Alternativas & Grandes & Medianas & $\begin{array}{l}\text { Micro y } \\
\text { Pequeñas }\end{array}$ & Promedio \\
\hline $\begin{array}{l}\text { Permite reconocer que no todos los } \\
\text { medios justifican los fines } \\
\text { Garantiza el cumplimiento de las }\end{array}$ & 3.7 & 2.8 & 3.9 & 3.5 \\
\hline leyes & 2.4 & 2.1 & 1.4 & 2.0 \\
\hline $\begin{array}{l}\text { Ayuda a la empresa en la definición } \\
\text { de acciones de voluntariado }\end{array}$ & 2.1 & 1.7 & 1.7 & 1.8 \\
\hline $\begin{array}{l}\text { Permite tener una posición } \\
\text { competitiva fuerte }\end{array}$ & 1.8 & 3.4 & 3.0 & 2.7 \\
\hline
\end{tabular}

FUENTE: ENCUESTA A EMPRESARIOS DEL SECTOR AGROALIMENTARIO DE MÉXICO.

Sin embargo, al analizar el tema de la responsabilidad social en relación a los desperdicios de alimentos, la mayoría de los entrevistados dijo que la responsabilidad era una medida de desempeño social por medio de "comportamientos filantrópicos" (cuadro 8). Es decir, los resultados muestran la poca o nula asociación que invariablemente los tomadores de decisiones lograron establecer entre desempeño de la empresa y la tarea de reducir los desechos de productos. El alfa de Cronbach de 0.89 muestra la gran coincidencia que, en este sentido, tuvieron los entrevistados.

A pesar de ello, los resultados de la siguiente pregunta (presentados en el cuadro 9) muestran que la conciencia ambiental está presente en la mayoría de los entrevistados, quienes dijeron que es importante medir las pérdidas de alimentos que genera la empresa, ya que "pueden provocar daños al medio ambiente" (3.7) y "podría ayudar a la comunidad" (2.9). La consistencia en las respuestas arrojó un valor de 0.86 en el alfa de Cronbach. 
Cuadro 8. La responsabilidad social empresarial, en materia de desperdicios de alimentos, es una medida del desempeño social por medio de:

\begin{tabular}{lrrrr}
\hline & \multicolumn{3}{c}{ Empresas } \\
\hline Alternativas & Grandes & Medianas & $\begin{array}{c}\text { Micro y } \\
\text { Pequeñas }\end{array}$ & Promedio \\
\hline Comportamientos filantrópicos & 3.9 & 3.3 & 3.5 & 3.6 \\
$\begin{array}{l}\text { Rentabilidad } \\
\text { Respeto a las leyes }\end{array}$ & 1.4 & 3.2 & 3.2 & 2.6 \\
$\begin{array}{l}\text { Respeto a las costumbres } \\
\text { informales }\end{array}$ & 2.9 & 1.7 & 1.3 & 2.0 \\
\hline
\end{tabular}

FUENTE: ENCUESTA A EMPRESARIOS DEL SECTOR AGROALIMENTARIO DE MÉXICO.

Cuadro 9. Es importante medir las pérdidas de alimentos que genera mi empresa ya que:

\begin{tabular}{lrrrrr}
\hline & \multicolumn{3}{c}{ Empresas } \\
\hline Alternativas & Grandes & Medianas & $\begin{array}{c}\text { Micro y } \\
\text { Pequeñas }\end{array}$ & Promedio \\
\hline $\begin{array}{l}\text { Quizás tengo costos que no he observado } \\
\text { Quizás puedo contratar }\end{array}$ & 0.3 & 1.6 & 1.5 & 1.1 \\
trabajadores & & & & & \\
Pueden provocar daño al medio ambiente & 1.7 & 2.1 & 2.0 & 1.9 \\
Podría ayudar a la comunidad & 4.2 & 3.1 & 3.7 & 3.7 \\
\hline
\end{tabular}

FUENTE: ENCUESTA A EMPRESARIOS DEL SECTOR AGROALIMENTARIO DE MÉXICO.

Al cuestionar la razón para disminuir las pérdidas de alimentos en México, los empresarios entrevistados dijeron que "son signos de ineficiencia" (3.1) y "son pérdidas económicas" (3.0) (Cuadro 10); alfa de Cronbach de 0.88.

Cuadro 10. Las pérdidas y desperdicios de alimentos son indeseables ya que:

\begin{tabular}{lcccr}
\hline & \multicolumn{3}{c}{ Empresas } & \\
\hline Alternativas & Grandes & Medianas & $\begin{array}{c}\text { Micro y } \\
\text { Pequeñas }\end{array}$ & Promedio \\
\hline Son pérdidas económicas & 3.3 & 2.8 & 2.9 & 3.0 \\
Son signo de ineficiencia & 3.4 & 3.1 & 2.7 & 3.1 \\
Hay gente pobre & 2.2 & 2.2 & 1.3 & 1.9 \\
Nadie quiere más basura & 1.1 & 1.9 & 3.1 & 2.0 \\
\hline
\end{tabular}


FUENTE: ENCUESTA A EMPRESARIOS DEL SECTOR AGROALIMENTARIO DE MÉXICO.

Sin embargo, a pesar de que no parecía un objetivo estrictamente económico, cuando se les cuestionó sobre la razón de emprender acciones para disminuir pérdidas de alimentos, la mayoría respondió que era para “disminuir costos” (3.2) y "permitiría precios más bajos (2.6), mientras que "cuidaría el medio ambiente" apenas obtuvo una graduación de 2.4 (Ver cuadro 11). La pregunta tuvo gran consistencia con un alfa de Cronbach de 0.87 .

Cuadro 11. Es importante para mi empresa disminuir las pérdidas de alimentos ya que:

\begin{tabular}{lcccc}
\hline & \multicolumn{3}{c}{ Empresas } \\
\hline Alternativas & Grandes & Medianas & $\begin{array}{c}\text { Micro y } \\
\text { Pequeñas }\end{array}$ & Promedio \\
\hline Disminuye mis costos & 3.5 & 2.8 & 3.2 & 3.2 \\
Permitiría precios más bajos & 1.7 & 2.8 & 3.3 & 2.6 \\
Ayudaría a la comunidad & 1.4 & 2.3 & 1.7 & 1.8 \\
Cuidaría el medio ambiente & 3.4 & 2.1 & 1.8 & 2.4 \\
\hline
\end{tabular}

FUENTE: ENCUESTA A EMPRESARIOS DEL SECTOR AGROALIMENTARIO DE MÉXICO.

De igual forma, al cuestionar sobre si era necesario crear un departamento especial para atacar el problema, la mayoría dijo que lo haría ya que "lograría medir el impacto sobre los costos" (3.1) y "ayudaría a la sociedad" (2.9) (Cuadro 12).

Cuadro 12. Es importante crear un departamento contra las pérdidas de alimentos en la empresa ya que:

\begin{tabular}{lrrrr}
\hline & \multicolumn{3}{c}{ Empresas } & \\
\hline Alternativas & Grandes & Medianas & $\begin{array}{c}\text { Micro y } \\
\text { Pequeñas }\end{array}$ & Promedio \\
\hline Lograría medir el impacto sobre & & & & \\
mis costos & 4.0 & 2.1 & 3.1 & 3.1 \\
Ayudaría a la sociedad & 3.2 & 2.8 & 2.6 & 2.9 \\
Disminuiría el impacto ambiental & 2.1 & 3.4 & 2.0 & 2.5 \\
Aumentaría mis ganancias & 0.7 & 1.7 & 2.3 & 1.6 \\
\hline
\end{tabular}

FUENTE: ENCUESTA A EMPRESARIOS DEL SECTOR AGROALIMENTARIO DE MÉXICO. 
Los resultados de todas las preguntas del cuestionario tienen representatividad al nivel de $95 \%$ de confianza. Finalmente, al cuestionar sobre la razón por la cual muchas empresas no realizan cotidianamente acciones para disminuir las pérdidas y desperdicios de alimentos, la mayoría dijo que "la empresa no es responsable" (2.5) y "no afecta los costos" (2.4). Muchos de los entrevistados dijeron que ellos no podían apreciar ningún costo, ya que "los precios de los productos ya consideraban esas pérdidas" (Cuadro 13). Nuevamente, al aplicar la prueba del coeficiente alfa de Cronbach, se obtienen resultados consistentes (alfa superior a 0.82) tanto en el segundo grupo de preguntas, como en el ejercicio total (12 preguntas) y para los tres sectores empresariales. Así, los resultados de este cuestionario se consideran confiables.

Cuadro 13. Disminuir las pérdidas de alimentos no son mi objetivo ya que:

\begin{tabular}{lcccr}
\hline Alternativas & Grandes & Medianas & $\begin{array}{c}\text { Micro y } \\
\text { Pequeñas }\end{array}$ & Promedio \\
\hline El gobierno debe resolverlo & 0.8 & 2.1 & 3.4 & 2.1 \\
La empresa no es responsable & 2.2 & 2.8 & 2.6 & 2.5 \\
No afectan mis costos & 2.1 & 3.4 & 1.7 & 2.4 \\
Ninguna de las anteriores & 4.9 & 1.7 & 2.3 & 3.0 \\
\hline
\end{tabular}

FUENTE: ENCUESTA A EMPRESARIOS DEL SECTOR AGROALIMENTARIO DE MÉXICO.

\section{CONCLUSIONES}

Esta investigación aporta dos innovaciones. Por una parte desarrolla un índice de pérdidas y desperdicios de alimentos que se aplica al caso de México pero que puede ser calculado desde bases de datos adecuadas, a cualquier país del mundo. Con una metodología novedosa y con bases de datos, se construye un indicador robusto del grado de pérdidas para un grupo de 34 alimentos, obteniendo un índice de pérdidas globales de 
alrededor de 35\%. Estas pérdidas de alimentos en México representan, a precios de mercado, alrededor de 10 mil millones de dólares anuales.

Por otro lado, los resultados muestran también el insuficiente desarrollo de acciones empresariales para disminuir las pérdidas y desperdicios de alimentos. Se aplicó un instrumento de medición del compromiso social de las pequeñas, medianas y grandes empresas. En particular, el cuestionario aplicado a empresas permitió captar, por primera vez en México, el grado en que los empresarios están comprometidos con tareas y acciones cotidianas para atender uno de los problemas sociales clave del país: la pérdida de

alimentos que podrían ser aprovechados para atender una de las manifestaciones más dramáticas de la pobreza extrema: el hambre. Destaca el hecho de que una gran proporción (96\%) de las micro y pequeñas empresas de México no desarrollan, cotidianamente, acciones de responsabilidad social corporativa que impliquen el logro del objetivo de minimizar pérdidas de alimentos.

Proponemos, entonces, la tarea de reducir desechos de alimentos como una gran área de oportunidad para la Responsabilidad Social Corporativa en México. Se sugiere, no obstante, el desarrollo de otros instrumentos de captación de información. El acceso a los tomadores de decisiones en materia de responsabilidad social empresarial es complicado en México, solo facilitado por el acceso a funcionarios de la administración pública.

\section{REFERENCIAS}

AGUILAR, Gutiérrez Genaro. Desigualdad y Pobreza en México, ¿Son inevitables? UNAM, IPN y Miguel Angel Porrúa, 2000.

BARBIERI, JOSE C. Gestão Ambiental Empresarial: Conceitos, Modelos e Instrumentos. $3^{\text {a }}$ ed. São Paulo: Editora Saraiva, 2011.

BENHUR MATTIUZ, JF DURIGAN. Effect of mechanical injuries on firmness and color of'Paluma'and'Pedro sato'guavas. Revista Brasileira de Fruticultura, 2001. 
CAIXETA FILHO, J.V. Losses in the transportation of fruits and vegetables: A Brazilian case study. International Journal of logistics: Research and applications, v. 12, n. 3, 2009 .

CALABRESE A; COSTA R; MENICHINI T; ROSATI, F. Does corporate social responsibility hit the mark? A stakeholder oriented methodology for CSR assessment. Knowledge and Process Management, 20(2), 77-89, 2013.

CARROLL, A. B.; SHABANA, K. M. The business case for corporate social responsibility: a review of concepts, research and practice. International Journal of Management Reviews, 12(1), 85-105, 2010.

CHITARRA, M. I.; CHITARRA, A. B. Pós-colheita de frutos e hortaliças: fisiologia e manuseio. 2. ed. Lavras: UFLA, 2005.

DAVIS, K. Can business afford to ignore social responsibilities? California Management Review, 2(3), 70-76, 1960.

FAO. Food Losses and Waste in Latin America and the Caribbean. Bulletin $\mathrm{N}^{\circ}$ 2; FAO Regional Office for Latin America an the Caribbean, 2015.

FAO. Food wastage footprint. Impacts on natural resources. Rome, 2013.

FAO. Pérdidas y Desperdicio de Alimentos en el Mundo. Alcance, causas y prevención. Roma, 2012.

FERRELL, O. C.; GONZALEZ-PADRON, T. L.; HULT, G. T. M.; MAIGNAN, I. From market orientation to stakeholder orientation. Journal of Public Policy \& Marketing, 29(1), 93-96, 2010.

FRIEDMAN, M. Capitalism and Freedom. The University of Chicago Press: Chicago, 1962.

GARDBERG, N. A.; FOMBRUN, C. J. Corporate citizenship: Creating intangible assets across institutional environments. Academy of management Review, 31(2), 329-346, 2006. 
GENIER, C., STAMP, M. \& PFITZER, M. Corporate social responsibility for agro-industries development. In: C. Da Silva, D. Baker, A. Shepherd, C. Jenane and S. Miranda-da-Cruz (eds), Agro-industries for Development. Oxfordshire, UK: CABI, 2009.

GOOCH, Martin; FELFEL Abdel; MARENICK, Nic. Food waste in Canada: Opportunities to increase the competitiveness fo Canada's agri-food sector, while simultaneously improving the environment. Value Chain Management Centre: Canada, 2010 .

GUNDERS, Dana. Wasted: How America Is Losing Up to 40 Percent of Its Food to Fork to Landfill. Natural Resources Defense Council; Issue Paper, August 2012; IP: 12-06-B, 2012.

HARTMANN, Momika. Corporate social responsibility in the food sector. European Review of Agricultural Economics Vol 38 (3), pp. 297-324, 2011.

HERTOG, M. L. A.T. M. et al. The impact of biological variation on postharvest behaviour: The case of dynamic temperature conditions. Postharvest Biology and Technology, v. 43, p. 183-192, 2007.

HULT, G. T. M. Market-focused sustainability: "Market orientation plus!". Journal of the Academy of Marketing Science, 39(1), 1-6, 2011.

KUMMU, M.; de MOEL, H.; PORKKA, M. et al. Lost food, Wasted Resources: Global Food Supply Chain Losses and Their Impacts on Freshwater, Cropland, and Fertiliser Use. Science of the Total Environment, 438, pp.477-489, 2012.

LÁZARO, L.; GREMAUD, A. A responsabilidade social empresarial e sustentabilidade na América Latina: Brasil e México. Revista de Administração da Universidade Federal de Santa Maria, 2016

LIU, Gang. Food Losses and food waste in China: a first estimate. Discussion paper, june; Industrial Ecology Programme; Norwegian University of Science and Technology (NTNU), Norway, 2013.

MATTIUZ, B.; DURIGAN, J. F. Efeito de injúrias mecânicas na firmeza e coloração de goiabas das cultivares paluma e pedro sato. Revista Brasileira de Fruticultura, v. 23, n. 2, p. 277-281, 2001. 
ORLITZKY, Marc. The politics of corporate social responsibility or: why Milton Friedman has been right all along. Annals in Social Responsibility, Vol. 1 Issue:1, pp.5 $-29,2015$.

RADI, M.; MAHROUZ, M.; JAOUAD, A. Phenolic composition, browning susceptibility, and carotenoid content of several apricot cultivars at maturity. HortScience, Alexandria, v. 32, n. 6, p. 1087-1091. 1997.

REICH, Alexander H.; FOLEY, Jonathan A. Food Loss and Waste in the US: The Science Behind the Supply Chain. Working paper 1239, Food Policy Research Center, University of Minnesota, 2014.

SAMIM, W.; BANKS, N.H. Colour changes in bruised apple fruit tissue. New Zealand Journal of Crop and Horticultural Science, Wellington, v. 21, n. 4, p. 367-72, 1993.

SOUZA, R. M.; HENZ, G. P.; PEIXOTO, J. R. Incidência de injúrias mecânicas em raízes de mandioquinha-salsa na cadeia de pós-colheita. Hortic. Bras.,v.21, n.4, 2003.

STONER J. A. F.; FREEMAN, R. E. ADMINISTRAÇÃO. 5a ed. Rio de Janeiro: LTC, 1999.

TACHIZAWA, T. Gestão Ambiental e Responsabilidade Social Corporativa. Estratégias de Negócios Focadas na Realidade Brasileira. 2a Edição, São Paulo: Editora Atlas, 2004.

VIVES, A. El papel de la RSE en América Latina: Diferente al de Europa? 2008.

ZHAO, WEN. Corporate Social Responsibility in the Energy Industry: A Content Analysis of Leading Energy Companies' Websites. Electronic Theses, Treatises and Dissertations. Paper 9505, 2015.

Recebido em: 14/10/2018 Aprovado em: 15/06/2019 Publicado em: 09/10/2019 